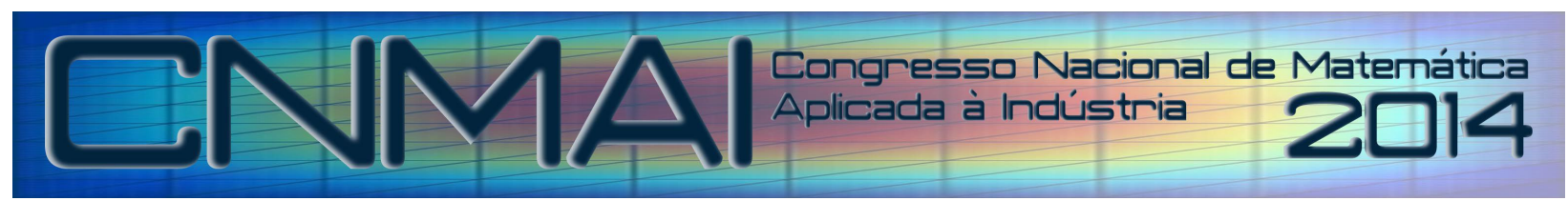

18 a 21 de novembro de 2014, Caldas Novas - Goiás

\title{
SIMULAÇÃO COMPUTACIONAL POR ELEMENTOS FINITOS E VALIDAÇÃO EXPERIMENTAL DE TUBOS DE PVC SOLICITADOS À FLEXÃO
}

\author{
Leandro Rodrigues da Silva Souza, leandrorodrigues.s@gmail.com \\ Núbia Saad dos Santos, nubia@ufu.br ${ }^{2}$ \\ Clezidan Núcio Pereira, cnucio@ibest.com.br ${ }^{3}$ \\ Vaston Gonçalves da Costa, vaston@gmail.com ${ }^{4}$ \\ Marcelo Henrique Stoppa, mhstoppa@gmail.com ${ }^{5}$
}

\author{
${ }^{1}$ Universidade Federal de Goiás, Av. Dr. Lamartine Pinto de Avelar, 1120, Setor Universitário, Catalão-GO \\ ${ }^{2}$ Universidade Federal de Uberlandia, Av. João Naves de Ávila, 2121, Campus Santa Mônica, Uberlândia-MG \\ ${ }^{3}$ Universidade Federal de Goiás, Av. Dr. Lamartine Pinto de Avelar, 1120, Setor Universitário, Catalão-GO \\ ${ }^{4}$ Universidade Federal de Goiás, Av. Dr. Lamartine Pinto de Avelar, 1120, Setor Universitário, Catalão-GO
}

\begin{abstract}
Resumo: O Policloreto de Vinila (PVC) é um polímero com diversas possibilidades de aplicação, cuja utilização é crescente, em substituição a outros materiais. Um dos principais motivos deste crescimento é o seu baixo custo, se comparado ao emprego de materiais tradicionais. Contudo, os polímeros possuem propriedades mecânicas diferentes de materiais metálicos ou cerâmicos. Um polímero que vem sendo bastante empregado é o PVC, principalmente na produção de tubos destinados ao transporte de fluidos, notadamente a água fria, em instalações prediais. Buscando avaliar o emprego de tubos de PVC como componentes estruturais solicitados à flexão simples, este trabalho apresenta uma simulação numérica, por meio do Método de Elementos Finitos (MEF). Foram realizados ensaios de flexão no Sistema de Ensaios MTS, e seus resultados confrontados com os obtidos numericamente, por meio de ferramentas computacionais CAE e CAD, para a validação da análise numérica realizada. Constata-se o quão os tubos de PVC são bastantes resistentes à flexão, o que possibilita uma maior possibilidade de seu emprego para fins estruturais, além do seu uso corrente, de transporte de água fria. A partir deste estudo, tem-se maiores informações do comportamento estrutural de tubos de PVC, permitindo maior aplicabilidade deste elemento estrutural de baixo custo e de fácil aquisição e manuseio.
\end{abstract}

Palavras-chave: Método de Elementos Finitos, Simulação Computacional, Experimental, Flexão,

\section{INTRODUÇÃ̃o}

A principal aplicação do PVC no mercado brasileiro tem sido na construção civil, onde é utilizado para a confecção de tubos e conexões, porém pode ser usado também para outros fins, como em pisos e esquadrias, ou mesmo na indústria automobilística.

Quando comparado com polímeros projetados para finalidades específicas como, por exemplo, suportar calor ou resistir a esforços mecânicos, os tubos de PVC apresentam um custo menor, sendo, no Brasil, utilizado largamente na construção civil devido à boa resistência mecânica e durabilidade (Gonçalves, 2001).

Há diversas técnicas para se produzirem tubos de PVC, como por exemplo, a técnica que emprega orientação molecular, que produz tubos com elevada resistência à tração e ao impacto quando comparados a tubos obtidos por processos convencionais (Medeiros, 2013). Com relação aos processos de transformação do PVC, a extrusão possui a maior representatividade dentre as técnicas empregadas (Rodolfo JR., 2006).

Para o uso na construção civil, existem os tubos destinados à alta pressão interna de serviço, cujas características mecânicas também são reforçadas.

Neste trabalho são empregados tubos destinados à baixa pressão interna em serviço, soldável e utilizado na construção civil, de baixo custo e fácil aquisição em relação às diversas possibilidades e tecnologias existentes no mercado. 
É realizada uma análise experimental dos tubos, solicitamos à flexão simples, com aplicação de carga concentrada no meio do seu comprimento, a fim de se validar uma simulação numérica proposta, pelo método de elementos finitos, com o auxílio do software SolidWorks ${ }^{\circledR}$.

\section{AVALIAÇÃO EXPERIMENTAL}

Para a confecção d os corpos de prova utilizados nos ensaios foram empregados tubos de PVC com $50 \mathrm{~mm}$ de diâmetro, fabricados segundo a norma da Associação Brasileira de Normas Técnicas (ABNT) NBR 5648. Todos os corpos de prova utilizados possuem $30 \mathrm{~cm}$ de comprimento com espessura do tubo de 4,5 mm.

Os corpos de prova foram extraídos de tubos com diferentes datas de fabricação, em duas situações de tempo de existência: aproximadamente 2 meses e 5 anos, sendo estes últimos considerados velhos. Vale ressaltar que, apesar disso, os tubos velhos foram acondicionados em ambiente adequado, com boa ventilação, sem incidência direta de raios solares ou chuva sobre os mesmos, uma vez que estas condições podem influenciar ou interferir no processo de degradação dos materiais polímeros (Rodolfo Jr., 2007).

Os corpos de prova foram ensaiados à flexão (Fig. 1), em condição de apoio bi apoiada, realizada através de conexões soldadas nas extremidades dos tubos.
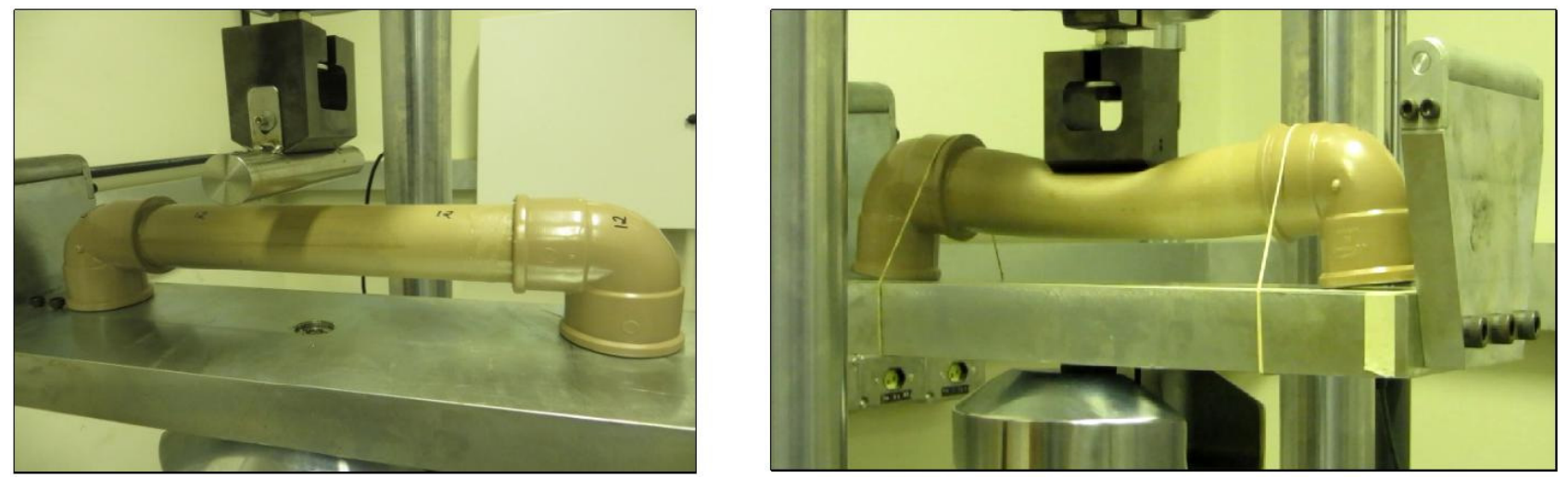

Figura 1. Vista do aparato montado para o ensaio de flexão do tubo, submetido à carga concentrada. Fonte: O Autor.

Foi aplicada uma força concentrada no meio do comprimento dos corpos de prova, de valor crescente, com taxa de carregamento de $2 \mathrm{~N} / \mathrm{min}$, até que os mesmos sofressem ruptura. Registra-se que a temperatura ambiente aquisitada durante os ensaios foi de $26^{\circ} \mathrm{C}$. Este ensaio visou à obtenção do limite de resistência à flexão, na fase elástica, e à medida do máximo deslocamento vertical ocorrido (no meio do comprimento do tubo), correspondente a este limite.

Para a realização deste ensaio, foi utilizado o Sistema de Ensaios MTS (modelo 793), disponível no LMEst (Laboratório de Mecânica de Estruturas Prof. José Eduardo Tannús Reis), da Faculdade de Engenharia Mecânica, da Universidade Federal de Uberlândia. Destaca-se que as conexões ficaram simplesmente apoiadas em uma base rígida de aço, conforme mostrado na Fig. 1.

\subsection{RESULTADOS E DISCUSSÕES}

Na Figura 2 tem-se os gráficos obtidos a partir dos ensaios, relacionando as forças aplicadas e os deslocamentos obtidos. Percebe-se que nos ensaios realizados utilizando os tubos velhos, com cinco anos de fabricação, não foi verificada alteração nos valores de resistência mecânica medida no limite da fase elástica, nem mesmo alguma mudança brusca ou considerável em seu modo de flexão em relação às demais amostras. No entanto, Moscow (2013) mostra evidências de redução da resistência mecânica para os tubos de PVC com mais de oito anos de fabricação.

Os diagramas força $\mathrm{x}$ deslocamento, ilustrados pela (Fig. 2) revelam a grande proximidade do comportamento mecânico do tubo de PVC solicitado à flexão, para os cinco corpos de prova ensaiados. 


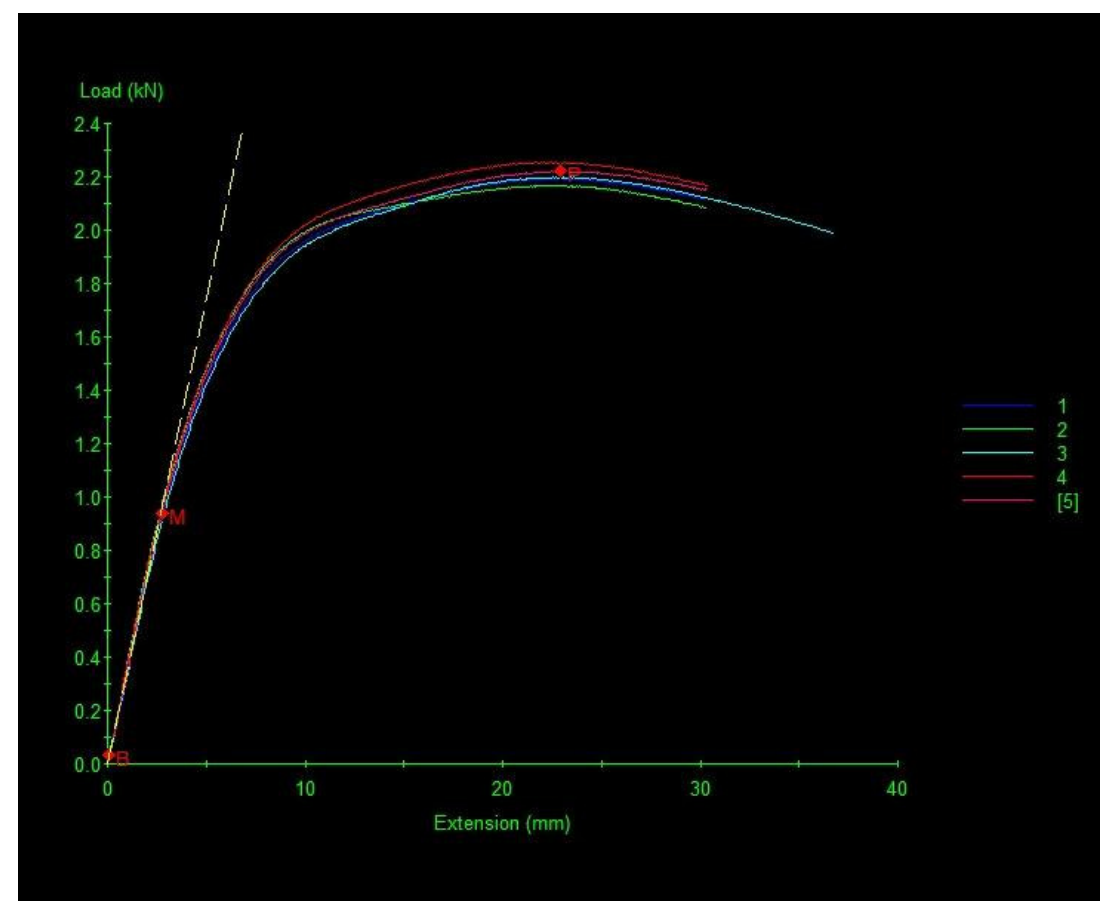

Figura 2. Diagramas gerados pelo programa pertencente ao Sistema de Ensaios MTS. Fonte: O Autor.

Os valores obtidos experimentalmente para a carga limite da fase elástica, bem como as medidas correspondentes dos deslocamentos para este nível de carga, estão mostrados na (Tab. 1). Os valores médios obtidos para ambas as variáveis foram de: $F_{\text {lim }}=0,925 \mathrm{kN}$ e $\delta_{\text {lim }}=2,719 \mathrm{~mm}$.

Tabela 1 - Resultados experimentais obtidos em ensaios de flexão.

\begin{tabular}{l|c|c|c|c|c|c}
\hline \multicolumn{1}{c|}{ Corpo de Prova } & $\begin{array}{c}\text { Corpo de } \\
\text { Prova -1 }\end{array}$ & $\begin{array}{c}\text { Corpo de } \\
\text { Prova -2 }\end{array}$ & $\begin{array}{c}\text { Corpo de } \\
\text { Prova -3 }\end{array}$ & $\begin{array}{c}\text { Corpo de } \\
\text { Prova - 4 }\end{array}$ & $\begin{array}{c}\text { Corpo de } \\
\text { Prova -5 }\end{array}$ & $\begin{array}{c}\text { Valor } \\
\text { Médio }\end{array}$ \\
\hline Carga Limite na Fase Elástica $(k N)$ & 0,913 & 0,902 & 0,929 & 0,930 & 0,939 & $\mathbf{0 , 9 2 2 6}$ \\
\hline $\begin{array}{l}\text { Deflexão do material sob Força } \\
\text { Limite }(\mathrm{mm})\end{array}$ & 2,729 & 2,543 & 2,829 & 2,652 & 2,756 & $\mathbf{2 , 7 1 8}$ \\
\hline
\end{tabular}

Inicialmente, acreditava que os tubos de maior idade, poderiam sofrer o colapso gerando estilhaços, no entanto, independentemente da idade do material, o mesmo se mostrou extremamente elástico e plástico, em ambas as fases do ensaio, sem se tornar quebradiço em alguma delas.

Revela-se que, durante os ensaios, os tubos não se romperam com a máxima deflexão considerada (medida além do limite da fase elástica, com situação visível de esmagamento bastante acentuado), nem geraram estilhaços, e os corpos de prova com diferentes idades apresentaram comportamentos semelhantes.

Outro aspecto interessante que foi constatado evidencia que mesmo na região de contato do tubo com a conexão soldada a este, não ocorreu colapso e nem estilhaço, apenas deformações plásticas.

\subsection{MATERIAIS E CONDIÇÕES DE CONTORNO}

Para validar com os resultados experimentais, foi realizada a modelagem computacional utilizando os recursos de CAD do SolidWorks@ considerando os mesmos parâmetros do ensaio experimental.

Para a simulação computacional são consideradas duas partes: o corpo de prova, representando o tubo de PVC, e o dispositivo para a aplicação da carga, em formato de um tubo de aço, semelhantes aos utilizados nos ensaios experimentais, podendo ambas as partes serem visualizadas na (Fig. 2) O Cilindro de aço representado pela (Fig. 2) tem por finalidade permitir a aplicação de uma força concentrada sobre o tubo. Sobre o corpo de prova em PVC é aplicado o material da biblioteca "Padrão de Materiais" disponibilizada pelo SolidWorks Simulation@ correspondente ao grupo de plásticos (que incluem o PVC rígido) aplicado para o tubo e do grupo de aço é definido para o cilindro de aço como sendo uma Liga de Aço Carbono podendo os parâmetros utilizados na definição das peças consideradas na simulação ser observados na (Tab. 2). 
Tabela 2 - Parâmetros dos materiais utilizados na simulação.

\begin{tabular}{|c|c|c|c|c|c|}
\hline \multicolumn{3}{|c|}{ Liga de Aço Carbono } & \multicolumn{3}{|c|}{ PVC Rígido Obtido Experimentalmente } \\
\hline Módulo elástico & $2 \mathrm{e}+011$ & $\mathrm{~N} / \mathrm{m}^{\wedge} 2$ & Módulo elástico & 1800 & $\mathrm{~N} / \mathrm{m}^{\wedge} 2$ \\
\hline Coeficiente de Poisson & 0.32 & N/A & Coeficiente de Poisson & 0,3825 & N/A \\
\hline Módulo de cisalhamento & $7,6 \mathrm{e}+010$ & $\mathrm{~N} / \mathrm{m}^{\wedge} 2$ & Módulo de cisalhamento & 866,7 & $\mathrm{~N} / \mathrm{m}^{\wedge} 2$ \\
\hline Massa específica & 7800 & $\mathrm{Kg} / \mathrm{m}^{\wedge} 3$ & Massa específica & 1300 & $\mathrm{Kg} / \mathrm{m}^{\wedge} 3$ \\
\hline Resistência à tração & 482549000 & $\mathrm{~N} / \mathrm{m}^{\wedge} 2$ & Resistência à tração & 40,7 & $\mathrm{~N} / \mathrm{m}^{\wedge} 2$ \\
\hline Resistência à compressão & & $\mathrm{N} / \mathrm{m}^{\wedge} 2$ & Resistência à compressão & & $\mathrm{N} / \mathrm{m}^{\wedge} 2$ \\
\hline Limite de escoamento & 248168000 & $\mathrm{~N} / \mathrm{m}^{\wedge} 2$ & Limite de escoamento & 1 & $\mathrm{~N} / \mathrm{m}^{\wedge} 2$ \\
\hline $\begin{array}{l}\text { Coeficiente de expansão } \\
\text { térmica }\end{array}$ & $1,2 \mathrm{e}-005$ & $\mathrm{~K}^{-1}$ & $\begin{array}{l}\text { Coeficiente de expansão } \\
\text { térmica }\end{array}$ & & K-1 \\
\hline Condutividade térmica & 30 & $\mathrm{~W} /(\mathrm{m} \cdot \mathrm{K})$ & Condutividade térmica & 0,147 & $\mathrm{~W} /(\mathrm{m} \cdot \mathrm{K})$ \\
\hline
\end{tabular}

A disposição e geometria das peças na modelagem computacional são realizadas conforme o experimento físico, nesse sentido, é de fundamental importância, que seja considerada a conexão das peças com uma geometria fixa entre as mesmas. Para o posicionamento e restrições quanto à disposição das peças foi empregado o recurso de montagem disponibilizado pelo SolidWorks@.

Destaca-se que, aqui, para que o módulo de elasticidade considerado na modelagem numérica fosse exatamente o real, ou seja, fidedigno ao ensaio experimental realizado, antes que se procedesse ao ensaio de flexão propriamente dito, os autores fizeram ensaios de tração em CPs de PVC também colhidos do mesmo lote de tubos aqui considerados. A partir dos ensaios de tração, foi possível se conhecer o valor médio do valor do módulo de elasticidade dos tubos em consideração: $\varepsilon$ médio = 1,8 GPa. Os demais parâmetros elásticos foram utilizados das bibliotecas disponíveis no pacote computacional SolidWorks Simulation@, conforme já mencionado.

Para as condições de contorno do modelo numérico foram considerados os parâmetros representados na (Fig. 3) sendo que a cor vermelha; o tubo tem seus apoios fixo e móvel, conforme (Fig. 3), representada pela Cor Verde, na cor magenta, a força aplicada no tubo pela área de contato deste com o atuador.

A disposição e geometria das peças na modelagem computacional são realizadas conforme o experimento físico, nesse sentido, é de fundamental importância, que seja considerada a conexão das peças com uma geometria fixa entre as mesmas. Para o posicionamento e restrições quanto à disposição das peças foi empregado o recurso de montagem disponibilizado pelo SolidWorks@.

Destaca-se que, aqui, para que o módulo de elasticidade considerado na modelagem numérica fosse exatamente o real, ou seja, fidedigno ao ensaio experimental realizado, antes que se procedesse ao ensaio de flexão propriamente dito, os autores fizeram ensaios de tração em CP's de PVC também colhidos do mesmo lote de tubos aqui considerados. A partir deste ensaio de tração, foi possível se conhecer o valor médio do valor do módulo de elasticidade dos tubos em consideração: $\varepsilon$ médio = 1,8 GPa. Os demais parâmetros elásticos foram utilizados das bibliotecas disponíveis no pacote computacional SolidWorks Simulation@, conforme já mencionado.

Para as condições de contorno do modelo numérico foram considerados os parâmetros representados na (Fig. 3) sendo que a cor vermelha; o tubo tem seus apoios fixo e móvel, conforme (Fig. 3), representada pela Cor Verde; na cor magenta, a força aplicada no tubo pela área de contato deste com o atuador.

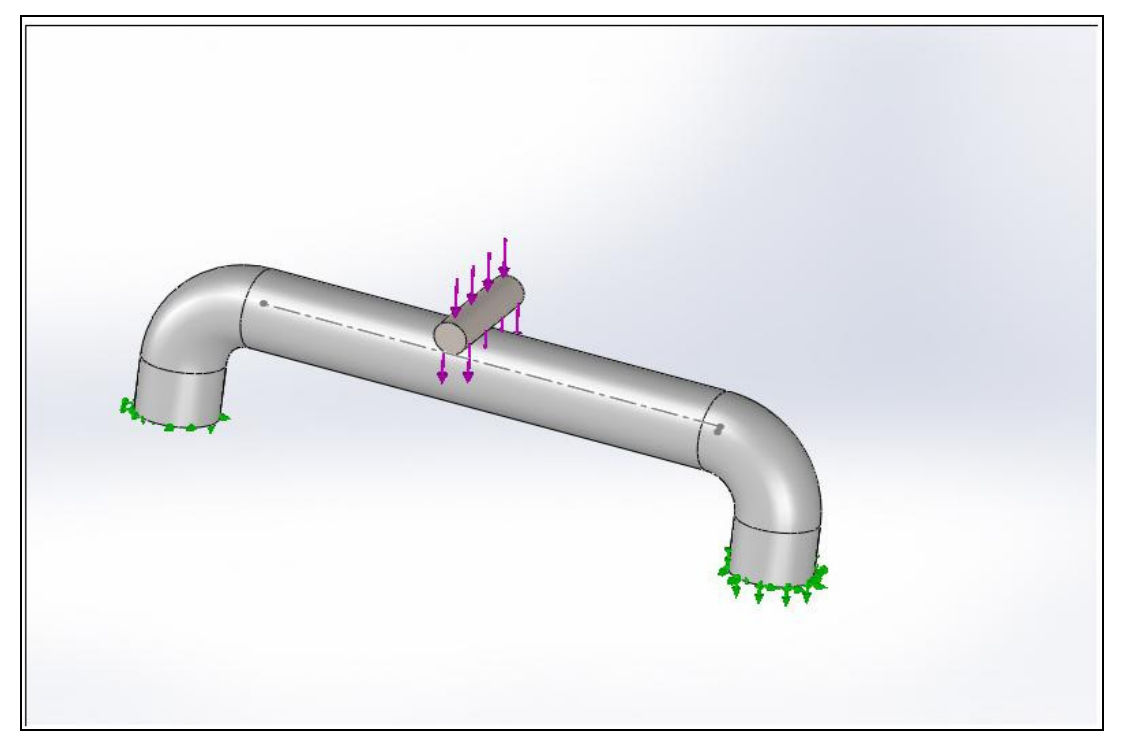

Figura 3. Condições de contorno utilizadas na simulação utilizando MEF. 
A força utilizada possui módulo $0,9226 \mathrm{kN}$, obtida experimentalmente (Tab.1). Os resultados dos ensaios foram obtidos com dois tipos de elementos do tipo casca para a composição da malha, conforme os mesmos parâmetros também analisados por Silva (2010), podendo ser visualizados pela (Fig. 4) suas características são:

- Draft: malha comumente utilizada para uma rápida avaliação, não sendo gerada com base em curvatura. Seu modo padrão gera uma malha utilizando elementos em forma de um triângulo linear, com três arestas.

- High: malha de alta qualidade, gerada com base em curvatura, onde os pontos jacobianos são o número de pontos a serem utilizados na distorção dos elementos tetraédricos. E seu modo padrão utiliza 6 nós, três por arestas, gerando elementos de casca triangulares parabólicos.

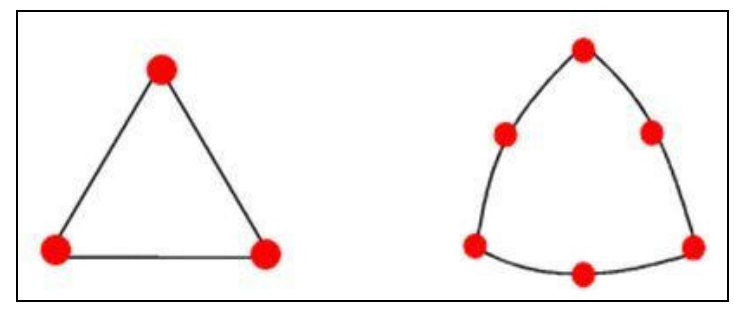

Figura 4. Elementos triangular parabólico. Fonte: SolidWorks@ (2014).

Ambos os tipos de elementos representados pela (Fig. 4) foram utilizados na geração de malha, classificada em dois grupos: de malha em modo Draft e em modo High, sendo uma das malhas utilizada nos ensaios representada na (Fig. 5) malha gerada em modo Draft com elementos de $8 \mathrm{~mm}$ triangulares.
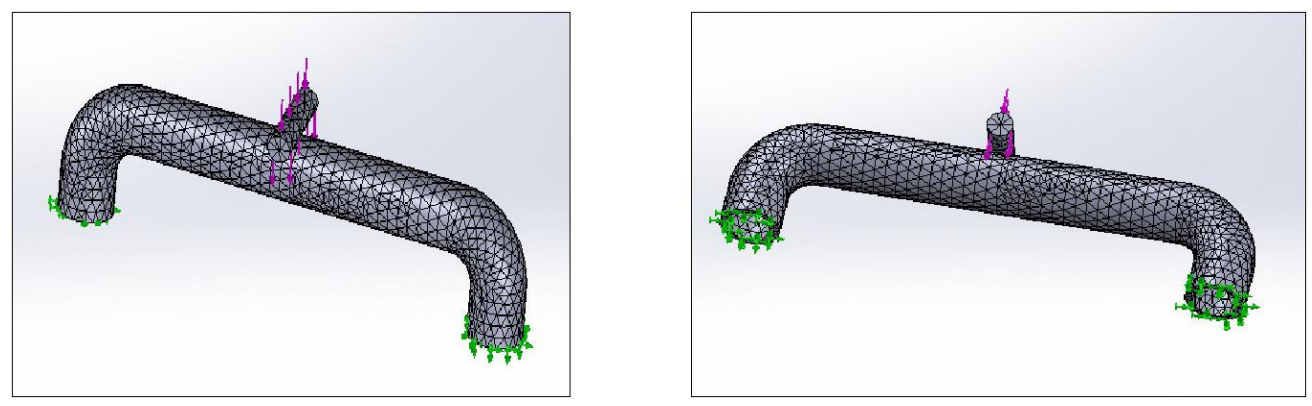

Figura 5. Elementos triangular linear malha com elemento de $8 \mathrm{~mm}$. Fonte: $\mathrm{O}$ autor.

Para avaliar a relevância do refinamento da malha, os valores para o deslocamento máximo e tensão máxima de von Mises na utilização de uma malha do tipo Draft são comparados, sob as mesmas condições, utilizando uma malha do tipo High.

A variação percentual comparativa entre malha do tipo Draft e malha em modo High é exibida na (Tab. 3).

Tabela 3. Resultados obtidos com elementos em modo Draft utilizando a definição de material obtida em ensaio experimental

\begin{tabular}{|c|c|c|c|c|c|c|}
\hline Ensaio & $\begin{array}{l}\text { Dimensão } \\
\text { do } \\
\text { Elemento } \\
(\mathrm{mm})\end{array}$ & $\begin{array}{c}\mathrm{N}^{\mathrm{o}} \text { de } \\
\text { Elementos }\end{array}$ & $\begin{array}{l}\text { Deslocamento } \\
\text { Máximo PVC } \\
\text { Ensaio (mm) }\end{array}$ & $\begin{array}{c}\text { Tensão } \\
\text { Máxima de } \\
\text { von Mises } \\
\text { PVC Ensaio } \\
(\mathrm{MPa})\end{array}$ & $\begin{array}{c}\text { \% Deslocamento } \\
\text { relativo à malha } \\
\text { PVC Ensaio } \\
\text { High }\end{array}$ & $\begin{array}{c}\% \text { Von Mises } \\
\text { relativo à Malha } \\
\text { High }\end{array}$ \\
\hline 1 & $8 \mathrm{~mm}$ & 7569 & 1,743 & 8,956 & $73 \%$ & $64,13 \%$ \\
\hline 2 & $7 \mathrm{~mm}$ & 9493 & 1,764 & 8450 & $70 \%$ & $64,90 \%$ \\
\hline 3 & $6 \mathrm{~mm}$ & 13944 & 1,808 & 9,742 & $74 \%$ & $66,52 \%$ \\
\hline 4 & $5 \mathrm{~mm}$ & 22078 & 1,889 & 11,41 & $75 \%$ & $69,50 \%$ \\
\hline 5 & $4 \mathrm{~mm}$ & 47797 & 1,970 & 11,95 & $79 \%$ & $72,48 \%$ \\
\hline 6 & $3 \mathrm{~mm}$ & 97576 & 2,134 & 117,8 & $85 \%$ & $78,51 \%$ \\
\hline 7 & $2 \mathrm{~mm}$ & 310473 & 2,2622 & 29,21 & $89 \%$ & $83,23 \%$ \\
\hline
\end{tabular}


Para a malha considerada High utilizando 4 pontos jacobianos, não foi observada mudança significativa se analisada pela variação do tamanho do elemento, para dimensões inferiores a $4 \mathrm{~mm}$, o que pode ser observado na (Tab. 4), a partir do ensaio 5. A partir de tal valor apenas a variação no tamanho do elemento já não é representativa para a convergência tanto do deslocamento quanto da tensão máxima de von Mises.

Utilizando a malha em modo High é realizada a comparação dos valores obtidos para o deslocamento em relação ao experimento físico, sendo utilizada para a comparação do deslocamento padrão a média obtida a partir da (Tab. 1).

Tabela 4. Resultados obtidos com ele mentos em modo High utilizando a definição de material obtida em ensaio experimental

\begin{tabular}{c|c|r|r|r|c}
\hline Ensaio & $\begin{array}{c}\text { Dimensão do } \\
\text { Elemento }(\mathrm{mm})\end{array}$ & $\begin{array}{c}\mathrm{N}^{\mathbf{o}} \text { de } \\
\text { Elementos }\end{array}$ & $\begin{array}{c}\text { Deslocamento } \\
\text { Máximo (mm) }\end{array}$ & $\begin{array}{c}\text { Tensão Máxima } \\
\text { von Mises(MPa) }\end{array}$ & $\begin{array}{c}\text { \% Deslocamento em } \\
\text { relação ao Experimento }\end{array}$ \\
\hline 1 & $8 \mathrm{~mm}$ & 6779 & 2,381 & 23,63 & $\mathbf{8 7 , 6 0 \%}$ \\
\hline 2 & $7 \mathrm{~mm}$ & 12756 & 2,668 & 30,03 & $\mathbf{9 8 , 1 6 \%}$ \\
\hline 3 & $6 \mathrm{~mm}$ & 17059 & 2,505 & 38,11 & $\mathbf{9 2 , 1 6 \%}$ \\
\hline 4 & $5 \mathrm{~mm}$ & 23346 & 2,510 & 38,21 & $\mathbf{9 2 , 3 5 \%}$ \\
\hline 5 & $4 \mathrm{~mm}$ & 49840 & 2,505 & 46,59 & $\mathbf{9 2 , 1 6 \%}$ \\
\hline 6 & $3 \mathrm{~mm}$ & 148434 & 2,599 & 55,61 & $\mathbf{9 5 , 6 2 \%}$ \\
\hline 7 & $2 \mathrm{~mm}$ & 310882 & 2,564 & $\mathbf{8 7 , 6 0 \%}$ \\
\hline
\end{tabular}

Dentre os ensaios executados aplicando duas configurações diferentes para gerar a malha, a melhor aproximação dos resultados ocorreu na utilização da malha High, onde a deformação máxima obtida via simulação computacional atinge $98,16 \%$ dos valores reais obtidos experimentalmente (Tab. 4) o comparativo entre os valores são representados pela (Fig. 6).

À medida que o tamanho do elemento utilizado para geração da malha tem suas dimensões reduzidas para cada malha Draft e High têm-se um comportamento especifico. Para o modo Draft à medida que o tamanho do elemento é reduzido é observada uma melhor aproximação da distensão em relação ao valor anteriormente obtido com um elemento de maiores dimensões.

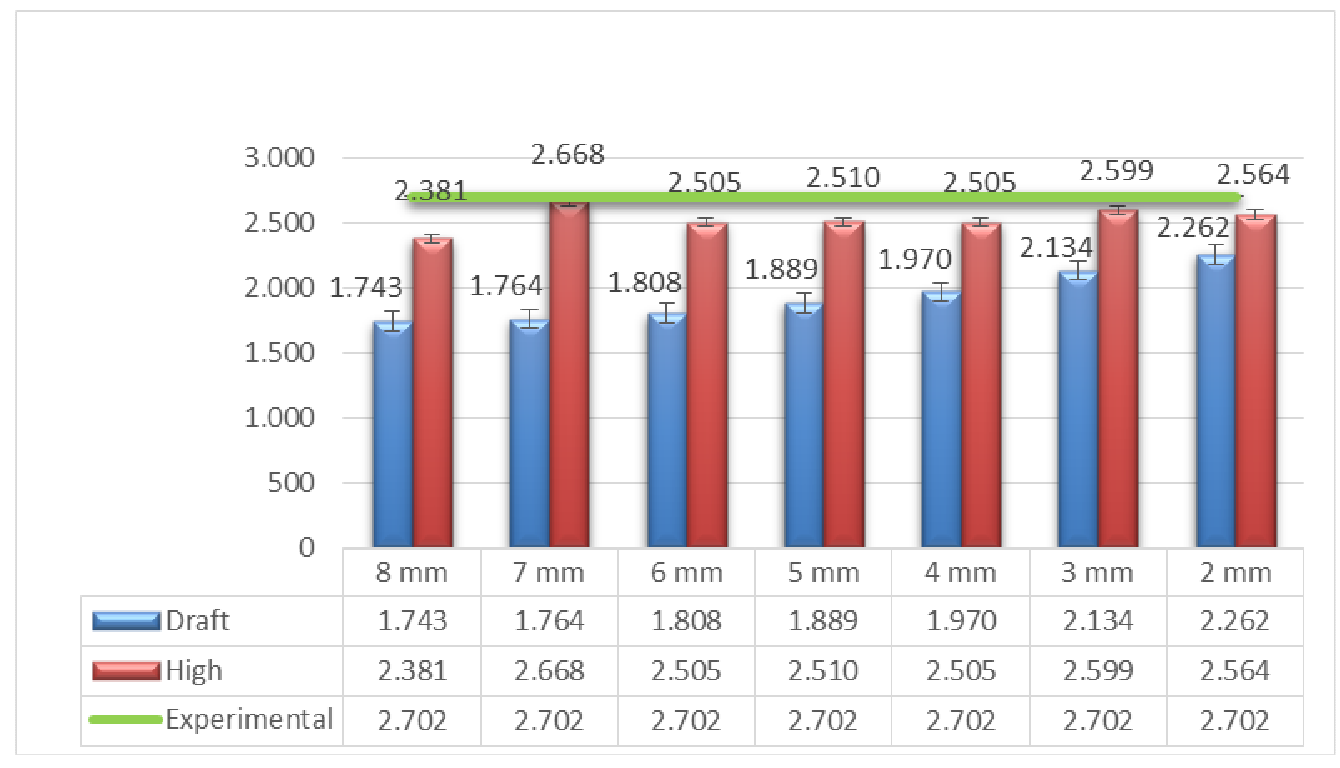

Figura 6. Deslocamento devido variação do tamanho do elemento da malha sob força constante. Fonte: Elaborada pelo autor.

Já para a malha do tipo High a redução no tamanho do elemento não irá produz necessariamente valores melhores que valores obtidos com o tamanho do elemento em maiores dimensões o que é visualizado na (Fig. 7). 


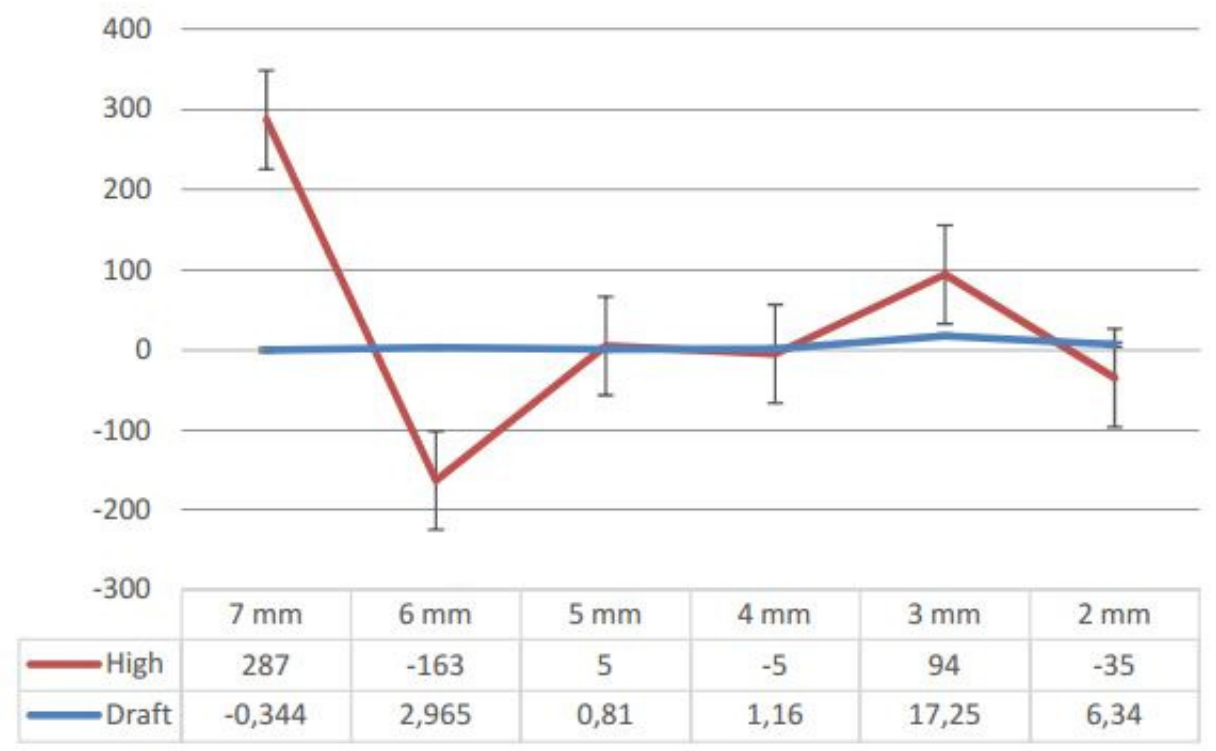

Figura 7. Comportamento da distensão frente à redução do elemento em relação ao valor anteriormente obtido com o elemento em maior tamanho. Fonte: $O$ autor.

Diante dos resultados foi empregado o controle da malha apenas para a região do tubo que é perpendicular à aplicação da força com o menor tamanho de elemento suportado pela geração de malha do SolidWorks@, obtendo-se o deslocamento representado pela (Fig. 9) e a tensão máxima de von Mises pela (Fig. 8). Com o valor de melhor convergência dentre todos os resultados de malha aplicados, quando comparado com o deslocamento experimental de 2,718 mm, é possível obter uma convergência de 98,16\% com 2,668 milímetros de deslocamento no tubo de PVC.

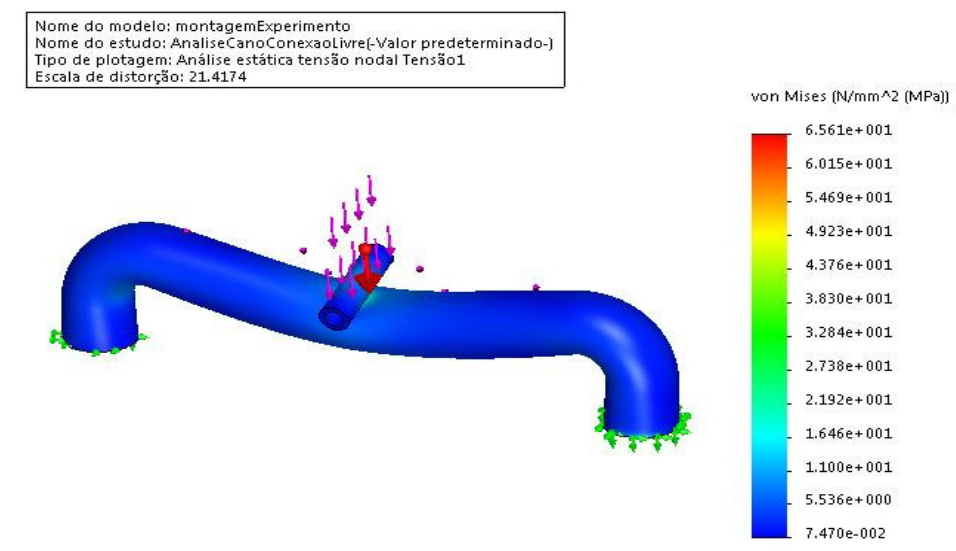

Figura 8. Tensão de von Mises obtida na melhor convergência em simulação. Fonte: O Autor.

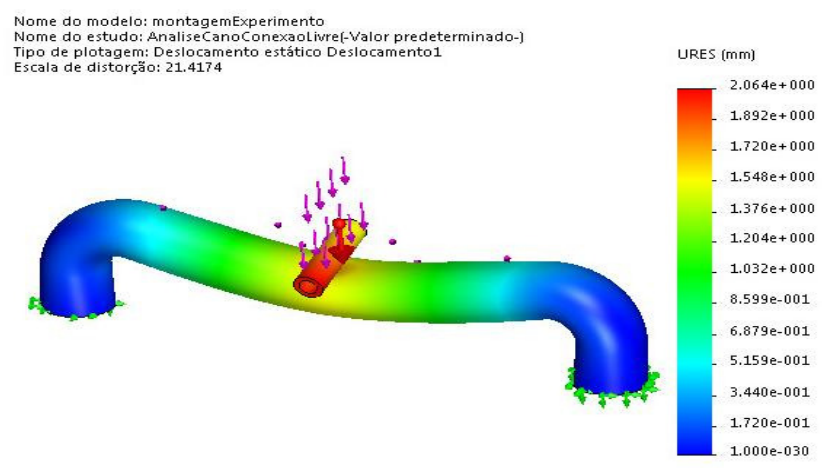

Figura 9. Deslocamento obtido na melhor convergência em simulação. Fonte: O Autor. 


\section{AGRADECIMENTOS}

Os autores agradecem ao Laboratório de Mecânica de Estruturas Prof. José Eduardo Tannús Reis (LMEstFEMEC-UFU) ao Instituto Nacional de Ciência e Tecnologia de Estruturas Inteligentes em Engenharia (INCT-EIE), pela realização dos ensaios bem como ao suporte financeiro parcial da CAPES, FAPEMIG, PPGGO/UFG/RC, FAPEG and $\mathrm{CNPq}$.

\section{CONLUSÕES}

Se Neste trabalho foi realizado o ensaio experimental de corpos de prova solicitados a Flexão e realizada a comparação dos valores da distensão frente à máxima força empregada ainda na fase elástica, sendo utilizada a simulação computacional como objeto de comparação, onde é empregada a variação da malha, no método numérico em que é aplicada a variação do tamanho do elemento que constitui a malha a aumento da quantidade de pontos dos elementos.

Pois, a utilização de uma malha correta tem fundamental importância para obter uma melhor convergência dos valores obtidos via método numérico, uma malha mais densa possui uma maior quantidade de nós, o que pode auxiliar na mensuração de deslocamentos em escalas menores, o que pode ser visualizado pelos resultados da variação do tamanho do elemento à medida que os elementos têm seu tamanho reduzido.

Por outro lado o aumento na densidade da malha aumenta o seu número de pontos e como consequência pode gerar um incremento considerável de tempo no processamento do método numérico. Diante dos dois grupos em que a malha sofre variação no tamanho do elemento ficou evidenciado que por menor que o mesmo possa ser reduzido não é o suficiente para aumentar a precisão do método numérico, que nas simulações realizadas se mostraram em um dado momento que ao reduzir o tamanho do elemento já não gerava influencia na distensão obtida via simulação.

Apesar do SolidWorks $\bigodot$ possuir valores considerados padrões para as propriedades de um dado material, tais valores do ponto de vista da resistência dos materiais são passiveis de variações para cada material uma vez que tanto o processo de fabricação, como a matéria prima, ou tecnologia utilizada, podem interferir em tais propriedades.

Assim é relevante a realização de outros experimentos que possibilitem a caracterização das propriedades do material para uma melhor convergência e conhecimento dos valores reais das distensões geradas para um dado material mesmo que as alterações sejam sutis, colaboram para permitir uma melhor convergência dos valores obtidos via simulação computacional.

Além de tais aspectos a validação da malha utilizada na simulação de forma experimental contribui para a adoção da malha a ser utilizada nos experimentos computacionais.

\section{REFERÊNCIAS}

Gonçalves, A., Almeida, S. F. M. e Neto, F. L. 2001.Comportamento de cilindros de carbono/epóxi submetidos a cargas compressivas axiais. Polímeros: Ciência e Tecnologia, vol. 11, n. 2, p.94-101.

Medeiros, Felipe Albuquerque; WIEBECK, Hélio. 2013. PVC Orientado: avaliação de processo de orientação e das propriedades mecânicas em função da razão de estiramento. Polímeros, São Carlos, v. 23, n. 5.

Miranda, Carlos Alberto Silva de. 2011. Simulação do processo de sopro de garrafas de poli (tereflalato de etileno) a partir de pré-formas disponíveis através do método de elementos finitos. Tese de doutorado Universidade Federal de Ouro Preto. Escola de Minas Rede Temática em Engenharia de Materiais.

Rodolfo JR., Antônio; MEI, Lúcia Helena I. 2007. Mecanismos de degradação e estabilização térmica do PVC: a review. Polímeros, São Carlos, v. 17, n. 3, Setembro.

Silva, Luís António Henrique. 2010. Avaliação Numérica do Comportamento Estrutural da Bancada de um Elevador.

Dissertação de mestrado em Engenharia Mecânica. Faculdade de Ciências e Tecnologia Universidade de Coimbra..

Schimits, Cristiane Salerno. 2002. Análise do comportamento estrutural de sistemas de drenagem viária constituídos por tubos plásticos flexíveis envolvidos com areia; Dissertação de mestrado Engenharia de Transportes; Escola Politécnica da Universidade de São Paulo.

Solidworks (Brasil). Web Help. 2014. Disponível em: <http://help.solidworks.com/2010/portuguesebrazilian/solidworks/cworks/legacyhelp/simulation/meshing_topics/id_shell_mesh.html>. Acesso em: jan. 2014.

\section{RESPONSABILIDADE AUTORAL}

Os autores são os únicos responsáveis pelo conteúdo deste trabalho. 


\title{
SIMULATION BY FINITE ELEMENT AND EXPERIMENTAL VALIDATION OF PVC PIPES BENDING UNDER DIFFERENT STRESS
}

\author{
Leandro Rodrigues da Silva Souza, leandrorodrigues.s@gmail.com ${ }^{1}$ \\ Núbia Saad dos Santos, nubia@ufu.br ${ }^{2}$ \\ Clezidan Núcio Pereira, cnucio@ibest.com.br ${ }^{3}$ \\ Vaston Gonçalves da Costa, vaston@gmail.com ${ }^{4}$ \\ Marcelo Henrique Stoppa, mhstoppa@gmail.com ${ }^{5}$
}

\begin{abstract}
${ }^{1}$ Federal University of Goiás, Av. Dr. Lamartine Pinto de Avelar, 1120, Setor Universitário, Catalão-GO
${ }^{2}$ Federal University of Uberlandia, Av. João Naves de Ávila, 2121, Campus Santa Mônica, Uberlândia-MG

${ }^{3}$ Federal University of Goiás, Av. Dr. Lamartine Pinto de Avelar, 1120, Setor Universitário, Catalão-GO

${ }^{4}$ Federal University of Goiás, Av. Dr. Lamartine Pinto de Avelar, 1120, Setor Universitário, Catalão-GO
\end{abstract}

\begin{abstract}
Polyvinyl Chloride (PVC) is used in various applications with the material being a polymer, and is growing as the use of polymers to substitute other materials in various application areas. One of the major aspects related to this growth is the low cost, relative to the use of traditional materials. However the mechanical properties of polymers differ considerably from metallic or ceramic materials, they exhibit very different behavior when subjected to stresses or deformations. A polymer used for a considerable time is Polyvinyl Chloride (PVC), mainly as pipes in building installations. However, these tubes are used almost exclusively for the transport of fluids, especially cold water. Trying to evaluate the use of these PVC pipes two different periods of manufacture and structural components, this paper presents computer simulations of these situations bending, subjected to different requests of force through the Finite Element Method (FEM). Because of the lack of material parameters, several experimental tests were performed in a traction machine, using PVC pipe samples used in building systems of cold water. Obtained the actual parameters displays a comparison of the experimental results and the simulation by finite element method (FEM), using CAD and CAE software tools in bending situations. Faced with this scenario is analyzed how sturdy are the PVC pipes to bending, for aiding from the understanding of mechanical behavior of a material the use of low cost, easy formability, compared to its restrictions be applied to other business scenarios
\end{abstract}

Keywords: Finite Element Method 1, Computer Simulations 2, Stress 3, PVC 4

The authors thank the Laboratory of Mechanics of Structures Prof. José Eduardo Reis Tannús (LMEst-FEMECUFU) at the National Institute of Science and Technology of Smart Structures in Engineering (INCT-EIS) for the tests as well as the authors gratefully acknowledge the financial support by CAPES, FAPEMIG, PPGGO/UFG/RC, FAPEG and CNPq. 